\title{
Expression analysis of fatty acid biosynthetic pathway genes during interactions of oil palm (Elaeis guineensis Jacq.) with the pathogenic Ganoderma boninense and symbiotic Trichoderma harzianum funga
}

\begin{abstract}
The fatty acid (FA) signaling pathway is emerging as an important mechanism in plant responses during interactions with microbial organisms. For a comprehensive evaluation of key FA biosynthetic pathway genes during interactions of oil palm (Elaeis guineensis Jacq.) with the pathogenic Ganoderma boninense and symbiotic Trichoderma harzianum fungal organisms, a lane-based array analysis of gene expression in artificially inoculated oil palm seedlings was performed. The results obtained demonstrated that acetyl-CoA carboxylase (ACC), 6-ketoacyl-ACP synthases (KAS) II and III, palmitoyl-ACP thioesterase (PTE), oleoyl-ACP thioesterase (OTE) and glycerol-3-phosphate acyltransferase (ACT) showed identical responses in root and leaf tissues for the same fungi. The expression of these genes was up-regulated in both root and leaf tissues at 21 days post-inoculation (dpi) during interaction of oil palm with G. boninense. Thereafter, production of physical symptoms occurred at 42 and 63 dpi concomitantly with suppression of expression of these genes. An increase in the expression level of these genes was observed in both tissues at $3 i ̈ 63 \mathrm{dpi}$, which correlated with the colonization of roots and promotion of plant growth by $\mathrm{T}$. harzianum. These data suggest that FA biosynthetic pathway genes are involved in the defense response of oil palm to infection. Identical plant responses by FA biosynthetic pathway genes may lead to enhanced resistance against $\mathrm{G}$. boninense and could be a useful marker to contribute towards early detection of infection. The distinct expression profile during symbiotic interaction demonstrated its role in plant resistance mechanisms and growth promotion by T. harzianum.
\end{abstract}

Keyword: Elaeis guineensis Jacq; Fatty acid biosynthetic pathway; Ganoderma boninense; Trichoderma harzianum 